\title{
Phenotypic characterization and familial risk in hyperplastic polyposis syndrome
}

October 2013, Vol. 48, No. 10 , Pages 1166-1172 (doi:10.3109/00365521.2013.830329)

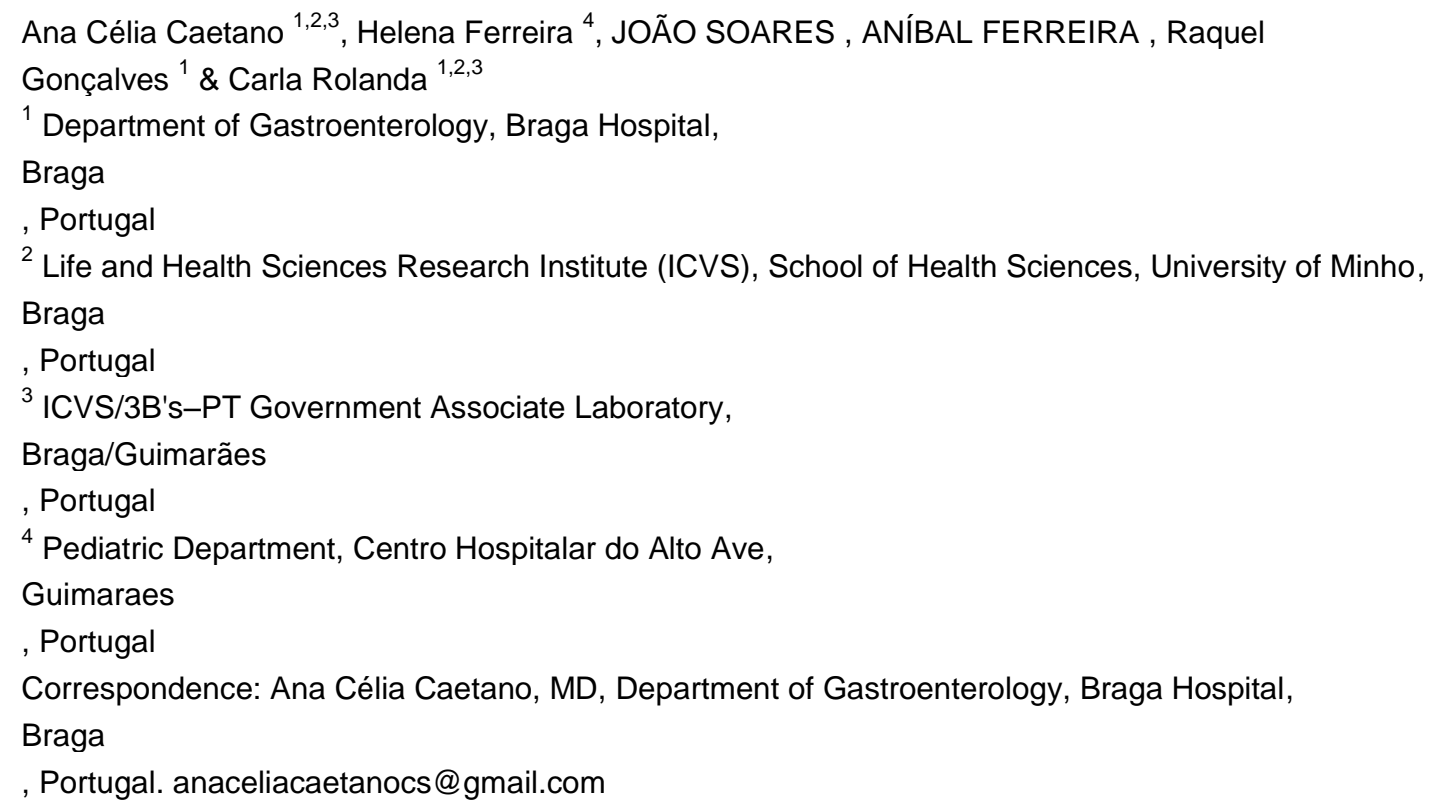

\section{Abstract}

Background. Hyperplastic polyposis syndrome (HPS) is a rare condition characterized by numerous hyperplastic polyps (HP) with a pancolonic distribution. Genetic and environmental factors, including smoking, may be responsible for phenotypic differences. Objective. To characterize HPS patients' phenotype and to determine HPS risk and colorectal cancer (CRC) risk in the first-degree relatives (FDRs). Patients and methods. Eight HPS patients were followed at our Gastroenterology Department (2008-2012). The data included (1) macroscopic and histological analysis of polyps, (2) demographic information about patients and their families and (3) colonoscopy results of FDR that accepted a screening exam. Results. Six of the eight index cases (ICs) had family history of CRC. Of the 24 FDRs screened, 5 were diagnosed with HPS. In our study, HPS and CRC prevalence in FDR was 625 and 9 times higher than the risk of the general population. Polyps over $10 \mathrm{~mm}$ were preferentially located in proximal colon $(p<0.001)$. Advanced polyps were larger $(p<0.001)$ than HP and more frequent in older patients $(p=$ $0.0054)$. Nonsmokers had smaller polyps $(p=0.037)$ preferentially in the proximal colon $(p=0.04)$ and a lower age at HPS diagnosis. Patients with CRC family history manifest HPS at an earlier age and patients whose relatives had CRC before 50 years had larger polyps $(p=0.0475)$. Smokers with $\mathrm{CRC}$ family history had larger polyps than nonsmokers $(p=0.048)$. Conclusion. Despite the small sample, the results reflect the phenotypic heterogeneity of HPS as well as the increased family risk of HPS and CRC. This study points out that CRC family history and smoking influence HPS expression.

Keywords: colorectal cancer, hyperplastic polyposis syndrome, phenotype, smoking 
Hyperplastic polyposis syndrome (HPS) is a rare disease with an estimated prevalence of 1:3000 in the general population [1]. Patients typically have multiple and large hyperplastic polyps (HPs) proximal to the sigmoid colon [2,3] . Different types of serrated polyps as HPs, sessile serrated adenomas (SSAs), traditional serrated adenomas (TSAs) and mixed polyps (MPs) are associated with HPS [3-7] . The SSAs, TSAs and MPs are considered advanced lesions [5,8]. Table I characterizes these polyps [8].

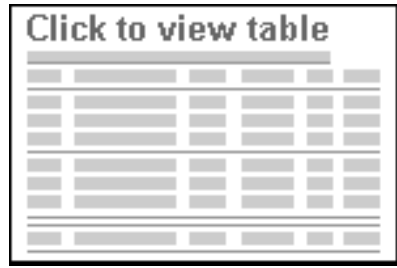

Table I. Morphological characteristics of serrated polyp types [8].

At least one of the following diagnostic criteria defined by Burt and Jass must be fulfilled: (1) a minimum of five HPs proximal to the sigmoid colon of which two being larger than $10 \mathrm{~mm}$; (2) any number of HPs proximal to the sigmoid colon in a patient with an FDR with diagnosis of HPS and (3) over 30 HPs distributed throughout the colon $[9,10]$.

An increased cancer risk in patients with multiple serrated polyps was reported $[2,10,11]$ especially if they are large, atypical, dysplastic or located proximally $[4,7,10,11]$. Carcinogenesis in serrated pathway involves a CpG island methylator phenotype (CIMP) and mutation of oncogene BRAF $[8,10,11]$. Although there are families with several affected members, suggesting a genetic predisposition $[2,3,5]$ no specific genes were identified so far. The pattern of inheritance seems to be autosomal recessive [5]. Besides genetic susceptibility, environmental factors probably determine tumor development. Various studies showed a strong association between smoking, serrated polyps and CRC with high microsatellite instability (MSI-H) [7,12-15].

Colonoscopy has some limitations in detecting serrated polyps because they are usually slightly elevated, pale and covered with mucus. This raised the interest in chromoendoscopy and virtual chromoendoscopy such as narrow-band imaging (NBI) to improve detection.

HPS patients should undergo colonoscopic surveillance with polypectomy, but so far the frequency of this surveillance was not defined $[14,16]$. HPS patients with FDR, who seem to be at higher risk for HPS and CCR than the general population, [2,17], should also be included in surveillance programs. Our aim is to determine the relative risk of HPS and CRC in FDR compared to our general population and to analyze the effect of genetics versus environment in HPS.

\section{Patients and methods}

\section{Study design}

We conducted a retrospective study in eight HPS patients, from the Gastroenterology Department of the Braga Hospital, Portugal, which were followed in outpatient setting between 2008 and 2012. These ICs were the starting point for family history and allowed us to contact the FDR for screening colonoscopy. Retrospective analysis of the ICs and their families was complemented with a prospective evaluation of the FDR. The study was approved by the Human Research Ethics Committee of the Braga Hospital. All participants signed their consent.

\section{ICs and family evaluation}


The majority of the ICs were referred for polypectomy by their family doctor, and HPS diagnosis was established after a complete (clinical, endoscopic and histological) evaluation. ICs did not meet phenotypic or familiar criteria for familial adenomatous polyposis, myh-associated polyposis or hereditary nonpolyposis colorectal cancer (CRC) although they were not submitted to genetic test. Data regarding age at diagnosis, follow-up, smoking status, total number, characteristics and location of polyps were collected. Smoking status was considered positive when active smoking habits were present. A family tree was traced for each IC. We then proposed a screening colonoscopy to all FDRs aged between 18 and 75 years, except for those who had a recent colonoscopy ( $<1$ year) whose reports were considered. Previous reports were not considered because a prospective evaluation was intended. Also, the majority of FDRs were beneath the age of CRC screening for average risk population and did not have any previous exam. More than 75 years and previous history of CRC were exclusion criteria. New cases of HPS in the FDR were classified as "screened cases" (SCs).

\section{Macroscopic and histological classification of the lesions}

Lesions were described as sessile, pedunculated or semipedunculated. Polyps' distribution was classified as predominantly proximal if $>50 \%$ of polyps were located in the proximal colon (from the cecum to the splenic angle), as predominantly distal if $>50 \%$ of polyps were located in the distal colon and the rectum (from the splenic angle to the dentate line) or as scattered if there was no predominance. Histological classification of serrated polyps follows the description of Table I; traditional adenomas (TAs) were classified into low- and high-grade dysplasias. Molecular analysis of the polyps was not performed (out of our objectives).

\section{HPS and CRC relative risk}

\section{Genetic and environmental effect on phenotypic expression}

Based on the available information, we calculated the familial risk of HPS and CRC development, in comparison with the general population. We assumed 1/3000 as the estimated prevalence of HPS in general population [2] and 29.1/3000 to be the CRC-estimated prevalence in Portuguese population [18]. HPS patients were also divided into groups: smokers versus nonsmokers and positive CRC family history positive versus negative CRC family history.

\section{Statistical analysis}

A descriptive analysis was performed. Continuous variables were evaluated using Mann-Whitney test and relations between variables were assessed for statistical significance using the chi-square $\left(x^{2}\right)$ or Fisher's exact test. The ratio between the HPS prevalence in the FDR and the general population was used to calculate the relative risk of the family members versus the general population. The same calculation was used to determine the $\mathrm{CRC}$ relative risk. Statistical analysis was performed using PASW Statistics 18 Chicago, Illinois SPSS 18.0 software, values equal to or less than 0.05 were considered statistically significant ( $p$-Value).

\section{Results \\ ICs and family evaluation}

Table II summarizes the demographic and familial aspects of the eight ICs. The majority were male (five cases) and the median diagnostic age was 43 years. In five patients, diagnosis was established after reference for polypectomy, whereas the remaining three cases were submitted to colonoscopy due to 
hematochezia, abdominal pain or standard screening. One patient had adenocarcinoma (in an adenomatous polyp) at the time of HPS diagnosis.

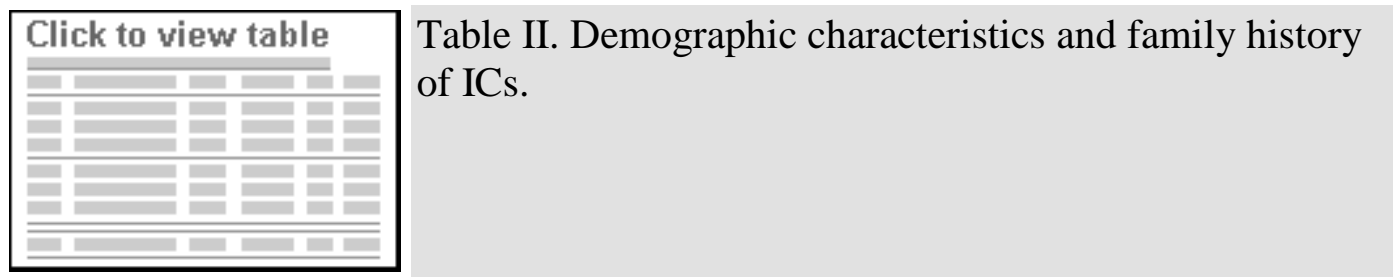

During the follow-up period (minimum 1 month and maximum 58 months), none of the patients developed $\mathrm{CRC}$. Colonoscopy was performed with a variable frequency (2-48 months) depending on findings, with a median of six polypectomies per exam. None of the patients reported a family history of HPS, but six patients had relatives with CRC.

From a total of 211 family members older than 18 years, 31 were excluded due to insufficient data. The information of the 180 remaining relatives was analyzed: 44 FDRs and 136 second-degree relatives (SDRs). We found a family history in six ICs (four FDRs and six SDRs with a median age of diagnosis of 45 years. We found a family history of other digestive cancers in two ICs (four cases-stomach, pancreas and liver), whereas family history of extradigestive cancers was present in four ICs (six cases-prostate, breast and brain).

From the 44 FDRs with more than 18 years old, 10 had already died, 2 had exclusion criteria and 8 refused to participate. Thus, 24 individuals ( 11 children, 8 siblings, 5 parents) were included in the study (17 females). The median age of children, siblings and parents was 28,42 and 56 years, respectively. Colonoscopy reports showed normal findings in 15 FDRs. Nine FDRs had polyps: five of them matched the diagnostic criteria for HPS and the other four (median age 36 years) had HP, but due to their distal location in the colon, HPS diagnosis was not set. The five SCs refer to three IC families, with earlier presentation in two families (third decade) and later presentation (sixth decade) in the other, as can be exemplified in Figure 1. No CRC was detected.

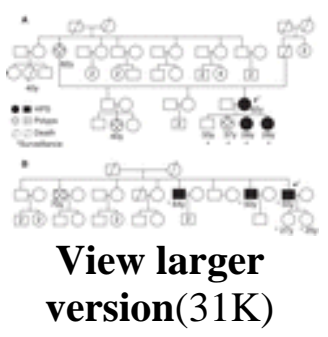
Figure 1. Two family trees with HPS: (A) earlier presentation and (B) later presentation.

\section{Macroscopic and histological classification of the lesions}

A total of 126 lesions were removed from ICs at diagnosis and during follow-up, a median total of 16 per patient (minimum 13 and maximum 60). The proximal colon was the predominant location in five cases. Pathological analysis showed that five patients had just HP, two patients had HP and TA, and only one patient had HP, TA and SSA. The largest polyps of each patient, usually between 5 and $10 \mathrm{~mm}$, were sessile, hyperplastic and located in the proximal colon. 
Considering the SC, a total of 22 lesions were evaluated and the median number per patient was 4 , essentially distributed in the distal colon (60\%). Three SCs had HP and two had HP and TA. The largest polyps of the SC (also $5-10 \mathrm{~mm}$ ) were preferentially located in the proximal colon.

Table III summarizes the important characteristics of the total number of lesions. Considering all lesions (148) of 13 HPS patients (IC plus SC), the median size was $7.2 \mathrm{~mm}$, the distribution was similar (75 in the proximal colon and 73 in the distal colon) and almost all were sessile (146).

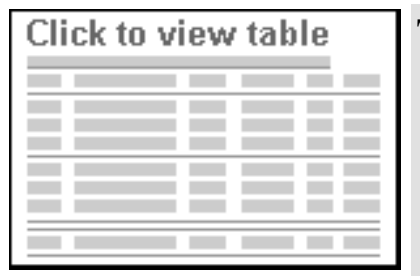

Table III. Total lesions (ICs plus SCs).

Comparing larger with smaller polyps, the former located preferentially in the proximal colon $(p<0.001$, $x^{2}$ test). Comparing HP polyps with histological more advanced lesions (SSA and TA), the latter had higher dimensions ( $6.95 \mathrm{~mm}$ vs. $9.33 \mathrm{~mm}, p<0.001$, Mann-Whitney). While HP were distributed uniformly throughout the colon, SSA and TA were mainly located in the proximal colon ( $p=0.089, x^{2}$ test). Advanced lesions were associated with older age $(p=0.0054$, Fisher.s exact test) and with a higher number of polyps-31 polyps versus 17 polyps in patients who only had HP ( $p=0.0715$, Mann-Whitney).

\section{HPS and CRC relative family risk}

Relative risk for developing HPS and CRC, respectively, is 625 and 9 times higher for the FDR than for the general population.

\section{Genetic and environmental effect on phenotypic expression}

Comparison of HPS prevalence in smokers and nonsmokers (Table IV) showed that smokers had significantly larger ( $p=0.037$, Mann-Whitney) and more advanced lesions ( $p=0.0197, \mathrm{X}^{2}$ test). There is a tendency among nonsmokers to develop HPS at younger age and to have lesions with proximal distribution. Although CRC family history appeared to be more relevant in nonsmokers, this difference was not statistically significant. The total number of polyps was also not significantly different between smokers and nonsmokers.

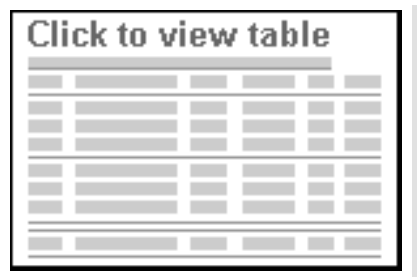

Table IV. Comparative analysis between smokers and nonsmokers.

Taking into account CRC family history, univariate analysis demonstrated that HPS appeared at an earlier age in individuals with $C R C$ family history compared to those without $C R C$ family history -29 versus 56 years-but this difference did not reach statistical significance ( $p=0.091$, Mann-Whitney). Patients whose parents had CRC before the age of 50 years had also significantly larger polyps than those whose relatives had CRC diagnosed after that age ( $p=0.0475$, Mann-Whitney). Similarly, among patients with CRC family history, smokers had larger polyps than nonsmokers ( $p=0.048$, Mann-Whitney). 


\section{Discussion}

An alternative sequence for CRC-the serrated pathway [12,19,20]-is widely accepted and stratification of serrated-risk lesions became an important issue [16]. With the expansion of knowledge in this area, the diagnostic criteria proposed by Jass and Burt will most likely be redefined [21]. Although HPS and CRC tend to run in families, there are just few studies proving this association $[2,22]$.

It is the rational of our intent to contribute to this discussion, recognizing, however, the limitations of our sample size. A multicenter, prospective study, instead of a single-center study, would probably be a best way to address this question.

Our results revealed a phenotypic heterogeneity in HPS patients not only in terms of number, size, histology and polyp distribution but also in terms of sex ratio, age at diagnosis and CRC family history.

Fifth and sixth decades are the usual age of diagnosis of HPS diagnosis $[4,6,17,22]$. However, similar to the findings of Chow et al. [3], the age of diagnosis in our IC was lower. The fact that the majority of our patients were submitted to colonoscopy before being 50 years old probably contributed to this finding. About sex ratio, studies are conflicting and we cannot exclude the existence of any bias.

The median number of polyps per patient varies in different studies: it was 16 in our study, which is in accordance with some authors [16,17] but is well below the average 90 polyps per patient documented by Ferrandez et al. [23] Our findings are probably related to patients' age and the presence of CRC family history; in fact, Buchanan et al. [20] pointed out the absence of CRC family history as an independent predictor of polyp density. Despite the predominance of small polyps, the presence of some larger lesions (high-risk lesions) is frequent. In our study, the analysis of larger lesions (28) showed that the majority were located in the proximal colon, in contrast to smaller polyps predominantly located in the rectum and the sigmoid, corroborating other publications [13,16]. One of the HPS characteristics is the simultaneous presence of different types of serrated and adenomatous polyps in the same patient $[3,16,17]$. However, we only found five cases of advanced lesions besides HP, which is probably due to the young age of our patients and the close follow-up with polypectomy. As demonstrated in previous studies, [3,17,22], HPs were the most prevalent lesions and SSAs corresponded only to $4.76 \%$ of all lesions removed. Since SSAs are considered the main precursor of CRC in HPS, its scarcity in our sample may explain the low number of CRC cases, in opposition to the works of Buchanan [24] or Lage et al. [17] in which $\sim 40 \%$ of patients had CRC. Interestingly, in our single case of adenocarcinoma, the tumor was grafted in TA.

An important finding is the association between preferential polyps' location and their histological grade. While HPs were distributed throughout the colon [4,17], the advanced polyps predominated in the proximal colon. These results emphasized the importance of polypectomy of all lesions found in the proximal colon, a therapeutic measure also suggested by other authors $[3,10]$. However, a distal preferential location in patients younger than 45 years was demonstrated-the earliest phenotypic form [3]. This information may justify a future stratification of colonoscopic surveillance taking into account lesions' distribution and patients' age.

We confirmed that HPS polyps are small, pale and sessile, which make their identification more difficult. For lesions $<10 \mathrm{~mm}$, the percentage of fault diagnosis may be as high as $23 \%$ [14]. However, in HPS, even small lesions can be histologically advanced explaining the "interval cancers" and the CRC risk even in individuals under endoscopic surveillance [14]. This reinforces the idea that HPS is underdiagnosed and probably a not so rare disease. 
HPS expression is apparently influenced by genetic and environmental factors. To our knowledge, no previous study analyzed the importance of having either CRC family history or smoking habits in the development of HPS and in disease course. We verified that our nonsmokers had an earlier diagnostic age, with smaller polyps located in the proximal colon. Simultaneously, the ones with a positive CRC family history expressed HPS also at an earlier age. Once smoking is a risk factor for serrated polyps, the age of diagnosis should be higher in nonsmokers than in smokers, but our data support the hypothesis of genetic load being stronger. In fact, nonsmokers, in our study, tend to have a higher familiar prevalence of CRC than smokers. We hypothesized that genetic factors (CRC family history) determine the age of onset, whereas environmental factors (smoking) function more as a modulator, probably promoting lesions' progression.

Fifty percent of our ICs (four) had FDR with CRC, which is in accordance with literature [4,17]. For an FDR, CRC risk was calculated as being 9 times higher than the estimated risk for the Portuguese general population. This value could have a bias due to our small sample, and the fact that some of our ICs were submitted to colonoscopy before 50 years, due to CRC family history. We could not conclude about the coexistence of HPS in those CRC relatives, probably because of clinical inadvertency to HPS in the past or even different HPS phenotypes in the same family.

Among the screened relative, there were five HPS new cases between screened relatives, restricted to three families, making a prevalence rate of $20.83 \%$ among screened FDRs, which is 625 times higher than the estimated for the general population [1]. This value could constitute a bias as only 24 of our 44 FDRs participated in our study and also because our prospective evaluation of FDRs did not consider data from previous colonoscopies, even though this was the first exam for the majority (only five FDRs had age for standard screening).

We identified some FDRs younger than 30 years with HP in the distal colon; even though they did not meet HPS diagnostic criteria and were excluded as SC, we were not sure about its significance. In fact, HPS in younger patients was associated with distal HP and CRC. It was also verified that SCs tend to present HPS around the third decade. From our data, it is not possible to infer the age of onset and consequently the most suitable moment to start surveillance in FDR, but the literature advises that first colonoscopy should be performed 10 years earlier than the age of diagnosis in ICs [22].

We could not clarify what the inheritance pattern of this syndrome is. Pedigree A shows an earlier presentation and a dominant mode of inheritance, whereas pedigree $B$ presents a later presentation and a recessive mode of inheritance. However, since it seems not to affect equally all generations and to predominate among siblings, an autosomal recessive trait seems likely. However, some of FDRs in the subgroup of children (median age 28 years old) may develop polyps at an older age, which could interfere in this evaluation.

We conclude that HPS is a heterogeneous disease, associated with a high risk of progression to CRC and family history. These data justify a surveillance program distinct from those proposed to the general population. However, we still lack a lot of information regarding the inheritance pattern as well as follow-up and treatment. Meanwhile, a more cautious approach should be generally recommended, consisting in polypectomy of all identified lesions and a close follow-up, preferably with the use of chromoendoscopy or NBI. It became clear that FDR should be under surveillance programs. Although in this study two forms of "family presentation" (early and late presentations) seemed to exist, only with a growing awareness of clinical diagnosis and with expanded studies, we will be allowed to know whether this disease has different phenotypes or the heterogeneity of disease is due to different genotypes.

\section{Acknowledgements}


All the authors (Ana Célia Caetano, MD; Helena Ferreira, MD; Raquel Gonçalves, MD; Carla Rolanda, $\mathrm{MD}, \mathrm{PhD}$ ) contributed for conception, critical revision and submission of this article.

Declaration of interest: None of the authors have any conflict of interest or disclosures to prepare this article.

\section{References}

1. Lockett MJ, Atkin WS. Hyperplastic polyposis: prevalence and cancer risk. Gut 2001;48:A4; AB 011.

ISI

2. Boparai KS, Reitsma JB, Lemmens V, van Os TA, Mathus-Vliegen EM, Koornstra JJ, et al. Increased colorectal cancer risk in first-degree relatives of patients with hyperplastic polyposis syndrome. Gut 2010;59:1222-5.

CrossRef, ISI

3. Chow E, Lipton L, Lynch E, D'Souza R, Aragona C, Hodgkin L, et al. Hyperplastic polyposis syndrome: phenotypic presentations and the role of MBD4 and

MYH. Gastroenterology 2006;131:30-9.

CrossRef, ISI

4. Hyman $\mathrm{NH}$, Anderson $\mathrm{P}$, Blasyk H. Hyperplastic polyposis and the risk of colorectal cancer. Dis Colon Rectum 2004;47:2101-4.

CrossRef, ISI

5. Young J, Jass JR. The case for a genetic predisposition to serrated neoplasia in the colorectum: hypothesis and the review of the literature. Cancer Epidemiol Biomarkers Prev 2006;15:1778-84.

CrossRef, ISI

6. Chan A, Issa JP, Morris JS, Hamilton SR, Rashid A. Concordant CpG island methylation in hyperplastic polyposis. Am J Pathol 2002;160:529-36.

CrossRef, ISI

7. Huang C, O'Brien M, Yang S, Farraye FA. Hyperplastic polyps, serrated adenomas, and the serrated polyp neoplasia pathway. Am J Gastroenterol 2004;99:2242-55.

CrossRef, ISI

8. Rosty C, Parry S, Young JP. Serrated Polyposis: An Enigmatic Model of Colorectal Cancer Predisposition. Pathol Res Int 2011;2011:157073. 
9. Jass JR, Burt RW. Hyperplastic polyposis. In: Hamilton SR, Aaltonen LA. editors. WHO International classification of tumors: pathology and genetics of tumors of the digestive system. Lyon: Springer Verlin; 2000. pp 133-4.

10. Lynch PM. Hyperplastic polyposis: semantics, biology, and endoscopy. Gut 2010;59:1019-21.

CrossRef, ISI

11. Jass JR. Hyperplastic polyps of the colorectum-innocent or guilty? Dis Colon Rectum 2001;44:163-6.

CrossRef, ISI

12. Kambara T, Simms LA, Whitehall VL, Spring KJ, Wynter CV, Walsh MD, et al. BRAF mutation is associated with DNA methylation in serrated polyps and cancers of the colorectum. Gut 2004;53:1137-44.

CrossRef, ISI

13. Rashid A, Houlihan PS, Booker S, Petersen GM, Giardiello FM, Hamilton SR. Phenotypic and molecular characteristics of hyperplastic polyposis. Gastroenterology 2000;119:323-32.

CrossRef, ISI

14. Young J, Jenkins M, Parry S, Young B, Nancarrow D, English D, et al. Serrated pathway colorectal cancer in the population: genetic consideration. Gut 2007;56:1453-9.

CrossRef, ISI

15. Ji BT, Weissfeld J, Chow W, Huang WY, Schoen RE, Hayes RB. Tobacco smoking and colorectal hyperplastic and adenomatous polyps. Cancer Epidemiol Biomarkers Prev 2006;15:897-901.

CrossRef, ISI

16. Boparai KS, Mathus-Vliegen EM, Koornstra JJ, Nagengast FM, van Leerdam M, van Noesel CJ, et al. Increased colorectal cancer risk during follow-up in patients with hyperplastic polyposis syndrome: a multicentre cohort study. Gut 2010;59:1094-100.

CrossRef, ISI

17. Lage P, Cravo M, Sousa R, Chaves P, Salazar M, Fonseca R, et al. Management of Portuguese patients with hyperplastic polyposis and screening of at-risk first-degree relatives: a contribution for future guidelines based on a clinical study. Am J Gastroenterol 2004;99:1779-84.

CrossRef, ISI

18. Kanavos P, Schurer W, Owusuapenten C, Sullivan R. Colorectal cancer in Europe and Australia: Chalenges and opportunities for the future. LSE Health 2008.

19. Rosenberg D, Yang S, Pleau D, Greenspan EJ, Stevens RG, Rajan TV, et al. Mutations in BRAF and KRAS differentially distinguish serrated versus non-serrated hyperplastic aberrant crypt foci in humans. Cancer Res 2007;67(8):3551-4. 
CrossRef, ISI

20. Goldstein N. Small colonic microsatellite unstable adenocarcinomas and high-grade epithelial dysplasias in sessile serrated adenoma polypectomy specimens. Am J Pathol 2006;125:132-45.

ISI

21. Lazarus R, Juntilla $O$, Karttunen $T$, Mäkinen MJ. The risk of metachronous neoplasia in patients with serrated adenoma. Am J Clin Pathol 2005;123:349-59.

CrossRef, ISI

22. Leggett $B$, Whitehall V. Role of serrated pathway in colorectal cancer pathogenesis. Gastroenterology 2010;138:2088-100.

CrossRef, ISI

23. Férrandez A, Samowitz W, DiSario J, Burt RW. Phenotypic characteristics and risk of cancer development in hyperplastic polyposis: case series and literature review. Am J Gastroenterol 2004;99:2012-18.

CrossRef, ISI

24. Buchanan D, Sweet K, Drini M, Jenkins MA, Win AK, Gattas M, et al. Phenotypic diversity in patients with multiple serrated polyps: a genetics clinic study. Int J Colorectal Dis 2010;25:70312.

CrossRef, ISI

Read More: http://informahealthcare.com/doi/full/10.3109/00365521.2013.830329 